\title{
Midiendo la exposición del cambio climático en las ciudades mesoamericanas
}

\section{Measuring the exposure of climate change in Mesoamerican cities}

\author{
Olivera, Sazcha; Labra, Diana; García, Lucero; Heard, Christopher; \\ Editor Académico Prof. Dr. Ángel Sol-Sánchez
}

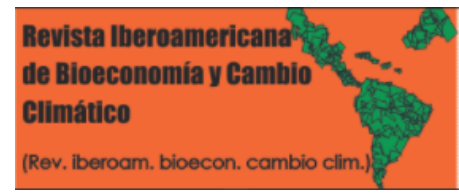

\author{
Sazcha Olivera \\ satzcha@msn.com \\ Universidad Autónoma Metropolitana, México \\ (iD) Diana Labra \\ diana.labramar@gmail.com \\ Universidad Autónoma Metropolitana, México \\ (D) Lucero García \\ dilucerof@gmail.com \\ Universidad Autónoma Metropolitana, México \\ Christopher Heard \\ cheard@prodigy.net.mx \\ Universidad Autónoma Metropolitana - Unidad \\ Cuajimalpa, Departamento de Teoría y Procesos del \\ Diseño, División de Ciencias de la Comunicación y \\ Diseño, México \\ Editor Académico Prof. Dr. Ángel Sol-Sánchez \\ Colegio de Postgraduados, México
}

\section{Revista Iberoamericana de Bioeconomía y Cambio Climático \\ Universidad Nacional Autónoma de Nicaragua, León, Nicaragua ISSN-e: 2410-7980 \\ Periodicidad: Semestral \\ vol. 6, núm. 11,2020 \\ czuniga@ev.unanleon.edu.ni}

Recepción: 17 Marzo 2020

Aprobación: 19 Julio 2020

\section{URL: http://portal.amelica.org/ameli \\ jatsRepo/394/3941758004/index.html}

Autor de correspondencia: satzcha@msn.com

Copyright (c) 2020 Revista Iberoamericana de Bioeconomia y Cambio Climático

\section{(i) () $\ominus$}

Esta obra está bajo una Licencia Creative Commons AtribuciónNoComercial-SinDerivar 4.0 Internacional.
Resumen: La presente investigación analiza la volatilidad de la precipitación y la temperatura en las ciudades mesoamericanas generadas por el cambio climático, utilizando como línea base la dinámica de las dos variables climáticas observadas mes a mes; entre 1971 a 1980 y 2001 a 2010 comparándolas con escenarios futuros de clima para los años 2061 a 2070. Las ciudades mesoamericanas utilizadas en el análisis que incluyen a las ciudades capitales de los estados del sudeste mexicano, Guatemala, El Salvador, Honduras y Belice. Medir la volatilidad de variables climáticas nos permite calificar si la distribución de lluvias y temperatura está por encima o por debajo de los rangos medios de la distribución histórica de estas variables. Entender la volatilidad climática, permite vislumbra una serie de escenarios de carácter técnico y político, y generar acciones concretas ante el cambio en las dinámicas climáticas de largo plazo y con ello anticiparse a los efectos de los eventos climáticos extremos presentes en una región. El método faculta la toma de decisiones en política pública, en particular en las áreas urbanas donde la tendencia a climas secos y cálidos genera una serie de consecuencias. Algunas consecuencias son i) la necesidad de diversificar las fuentes de agua potable y la generación hidroeléctrica, ii) los efectos de las rachas de calor en la calidad de vida de la población, iii) la calidad de sueño y productividad y en última instancia iv) el consumo de niveles de energía destinados a aire acondicionado o refrigeración superiores a los niveles actuales.

Palabras clave: Precipitación, Temperatura, Areas urbanas, Mesoamérica, Volatilidad, Eventos extremos.

Abstract: The present investigation analyzes the volatility of
precipitation and temperature in Mesoamerican cities generated
by climate change, using as a baseline the dynamics of the two
observed climatic variables month by month; between 1971 to
1980 and 2001 to 2010 , comparing them with future climate
scenarios for the years 2061 to 2070 . The Mesoamerican cities
used in the analysis include the capital cities of the states
of the Mexican southeast, Guatemala, El Salvador, Honduras,
and Belize. Measuring climatic variables' volatility allows us to
qualify if the distribution of rainfall and temperature is above or
below the average ranges of the historical distribution of these
variables. Understanding climate volatility provides a glimpse of
a series of technical and political scenarios and generates concrete 
actions in the face of changes in long-term climate dynamics. Thus, it anticipates the effects of extreme climate events present in a region. The method empowers decision-making in public policy, particularly in urban areas where the tendency to dry and hot climates generate a series of consequences. Some consequences are i) the need to diversify drinking water sources and hydroelectric generation, ii) the effects of heat spells on the quality of life of the population, iii) the quality of sleep and productivity, and ultimately iv ) consumption of energy levels for air conditioning or refrigeration higher than current levels.

Keywords: Precipitation, Temperature, Urban areas, Mesoamerica, Volatility, Extreme events.

\section{INTRODUCCIÓN}

Tanto la evidencia científica como empírica demuestra que el cambio climático es un problema real con impactos en todo el mundo. Eventos extremos como la perdida de glaciares, sequias, inundaciones e incendios hacen evidente que, como humanidad, estamos expuestos al cambio climático; en este sentido, medir los posibles efectos de este cambio nos permitirá adaptarnos como sociedad a los impactos esperados y generar políticas específicas para frenar los efectos de los cambios del clima (Martinez Gonzalez, 2015).

Las ciudades en Latinoamérica y en particular en Mesoamérica si bien no son importantes emisores de gases de efecto invernadero [GEI], desempeñan funciones cruciales en los procesos de adaptación ante el cambio climático global (Hardoy \& Lankao, 2011). Ello debido a la creciente urbanización de América Latina y en particular de México y Centro América. Las ciudades no solo son fuentes crecientes de gases de efecto invernadero a futuro, sino que también son puntos críticos de vulnerabilidad a inundaciones, sequias olas de calor y otros peligros que se espera se agraven por los acontecimientos de eventos climáticos extremos (Satterthwaite, 2009).

Uno de los aspectos primordiales para entender la relación entre las áreas urbanas y la ocurrencia de eventos climáticos y su volatilidad, de índole puntual o de tendencia de largo plazo, es el consumo de energía ya sea para el bombeo de agua potable, el manejo de plantas de tratamiento de aguas residuales, el transporte de mercancías (Olivera S. M., 2017), el manejo de aire acondicionado, calefacción y su relación con la construcción de infraestructura urbana resiliente y el diseño de bienes de vida de larga duración como edificios y casas (Heard y Olivera, 2013).

En este sentido, la presente investigación analiza la volatilidad de la precipitación y la temperatura en las ciudades mesoamericanas generadas por el cambio climático, utilizando como línea base la dinámica de las dos variables climáticas observadas mes a mes; la primera abarca de 1971 a 1980 y la segunda de 2001 a 2010 (Oglesby et al., 2016). Las ciudades mesoamericanas utilizadas en el análisis incluyen a las capitales de los Estados de México (Campeche, Chiapas, Guerrero, Oaxaca, Puebla, Quintana Roo, Tabasco, Veracruz y Yucatán), así como las ciudades capitales de Guatemala, El Salvador, Honduras y Belice. La volatilidad se entiende como la variabilidad de la precipitación o temperatura con respecto a su media en un periodo de tiempo determinado.

"La volatilidad es una medida de la intensidad de los cambios aleatorios o impredecibles en el comportamiento de un variable; en la representación gráfica de una serie histórica se asocia la volatilidad

\section{NotAS DE AUTOR}


con la amplitud de las fluctuaciones del comportamiento de la variable tanto es que se consideren en valor absoluto como en desviaciones alrededor de un valor medio" (Figlewski, 1997).

Medir la volatilidad de variables climáticas nos permite calificar si la distribución de lluvias y temperatura está por encima o por debajo de los rangos medios de la distribución histórica, para con ello distinguir si hay falta de lluvias en forma acumulada y no solo puntual. Al cuantificar la volatilidad se puede apreciar si una región tuvo lluvias o temperaturas por debajo de las medias esperadas o si los escenarios futuros presentan dinámicas por encima de la media histórica.

Entender la volatilidad vislumbra una serie de escenarios de carácter técnico y político para generar acciones concretas ante el cambio en las dinámicas climáticas de largo plazo y ver sinergia con los eventos climáticos extremos presentes en una región. Observar a la volatilidad en las localidades en periodos establecidos, permite realizar una proyección de la tendencia futura del cambio climático y con ello entender las consecuencias para la toma de decisiones.

La metodología seleccionada para el análisis de volatilidad utiliza el concepto de distancias estadísticas a partir del análisis de las medias históricas de las variables de análisis. El método fue aplicado para el desarrollo de índices de vulnerabilidad climática por el Instituto Mexicano de Competitividad [IMCO] (2012) y por Olivera (2019); este tiene como principal ventaja el uso de datos históricos de series cortas pudiendo desarrollar comparaciones incluso con series de 20 años atrás, siendo un método de carácter informativo, que usa categorías de información dándole a la metodología un carácter más de política pública que permite el desarrollo de índices o políticas de adaptación local que de una herramienta técnica a nivel estadístico (DARA, 2012)

El presente artículo se estructura de la siguiente manera: en primer lugar, se analiza la relación entre la variabilidad climática y las áreas urbanas, posteriormente se realiza un análisis de la volatilidad de variables climáticas a partir del estudio de series históricas de corta duración (menos de 50 años), en las ciudades mesoamericanas del norte de la región, que incluyen los Estados del sur de México, Guatemala, Belice, Honduras y el Salvador. Finalmente, finalmente se discute y concluye la relevancia de los resultados hallados con la aplicación de la metodología.

\section{EXPosición DE LA METODOLOGÍA}

Los efectos del cambio climático se pueden observar a través de la construcción de índices de tendencia de volatilidad; dilos cuales proporcionan información necesaria para capturar y manifestar la vulnerabilidad de cada área urbana analizada, en relación con la temperatura y precipitación como consecuencia del cambio climático.

En estadística descriptiva se utilizan múltiples métodos para el análisis de datos, en este caso usaremos el concepto de distancia como la diferencia entre dos objetos, es decir entre poblaciones, individuos, clima, etc. Estos conceptos han sido utilizados en muchos campos científicos, en este caso lo utilizaremos para analizar la distancia que hay en parámetros climáticos (precipitación y temperatura) entre dos periodos de tiempo. Donde la distancia compara los parámetros climáticos en función del cambio de la volatilidad de la variable y el cambio del promedio mensual de la variable de análisis (Loa, Cabos, Álvarez García, y Ruiz de Elvira, 2006). Los indicadores incluidos en este apartado describen cambios en los niveles y las volatilidades de la temperatura y la precipitación, registradas diariamente a lo largo de un mes. Lo que permite distinguir si la volatilidad y el promedio dentro del mes analizado ha sufrido un cambio, ya sea en incremento o en decremento.

Una diferencia sustancial de la metodología desarrollada por IMCO (2012) es el uso de datos a nivel de áreas urbanas y no a nivel municipal. Para la generación de datos a nivel área urbana se utilizó la base de datos histórica: NNRP que es parte de los datos RCCDP Data Download, Se analizaron tres periodos 1971-1980, 2001-2010 y con la base CCSM4_rcp85 Midcentury para las proyecciones futuras de clima 
se estudió 2061-2070 (REGIONAL CLIMATE CHANGE CONSORTIUMFOR LATIN AMERICA AND THE CARIBBEAN, 2020), para cada una de las áreas urbanas utilizando una resolución de 12 y $4 \mathrm{~km} 2$. Según Oglesby et al (2016) estas resoluciones permiten replicar y tener un ajuste mucho más adecuado a las características topográficas locales que se requieren para analizar ciudades y centros urbanos.

La comparación a través del tiempo se hace con base en el mes analizado. La agrupación de datos permite identificar el comportamiento, a lo largo de esos años, de las dos variables climáticas en un mes específico. Para comparar las variables de estos tres intervalos de tiempo se hizo el cálculo de la diferencia del promedio de la variable (temperatura y precipitación) para cada intervalo de tiempo.

$A:=$ Promedio del mes del periodo 1.

$B:=$ Promedio del mes del periodo 2.

$P_{D}:=$ Diferencia del mes entre dos periodos.

$P_{D}=B-A$

Utilizando la temperatura promedio mensual y la precipitación total media mensual desde 1971 hasta 1980 a partir de los datos anteriores se calcularon los siguientes estadísticos que arrojaran información por cada variable analizada:

- Desviación estándar para dos grupos de años: 1971-1980 y 2001-2010.

- Valor promedio para los mismos grupos de años: 1971-1980y 2001-2010.

Para hacer viable la comparación de los municipios y el valor de las variables, se buscó que los estadísticos reflejaran para cada mes lo siguiente:

- Cambio porcentual de la desviación estándar de la variable en el municipio (1971-1980 vs 2001-2010)

- Cambio porcentual del promedio de la variable en el municipio (1971-1980 vs 2001-2010)

El cambio porcentual nos dice la diferencia entre un valor pasado y uno presente en términos de un porcentaje del valor pasado. Este indicador evalúa la variabilidad de la precipitación y la temperatura en un mes determinado dentro de un intervalo de tiempo. Es calculado de la siguiente manera:

$C$ :=Desviación estándar del mes del periodo 1.

$D:=$ Desviación estándar del periodo 2 .

$\triangle$ Desv $:=$ Cambio porcentual de la desviación estándar.

Para la extracción de los datos se elaboró un programa en MATLAB que busca en la base de datos las coordenadas más cercanas a las del área urbana que se quiere estudiar para obtener información de ese punto.

$\Delta \operatorname{ses}-\left(\frac{p-c}{c}\right) \cdot 100$

Los estadísticos descritos anteriormente han sido calculados para dos grupos de años. Se utilizaron los años que abarcan de 1971 hasta 1980 para delimitar una serie de tiempo que funcionará como parámetro de control del clima, lo que permitió comparar contra un grupo de años que entregue información sobre las mismas variables,

pero en función a escenarios climáticos futuros. De esta manera se hacen evidentes los cambios porcentuales en nivel y variabilidad de los datos observados a lo largo de décadas en cada área urbana analizada. Secuencialmente se puede percibir el cambio en las variables climáticas durante la serie de tiempo analizada en comparación a los datos base. Cabe señalar que las pruebas estadísticas realizadas, por ejemplo, diferencia de medias o diferencia de desviaciones estándar, no validan ninguna hipótesis, ya que este trabajo de investigación pretende ofrecer información descriptiva de un fenómeno climatológico y visualizar una tendencia general de la volatilidad en función a la distancia de la variable de control y la serie de tiempo analizada.

El siguiente paso en la creación de indicadores consistió en seguir una tipología de volatilidad de la temperatura y la precipitación que permite clasificar a las áreas urbanas de acuerdo con la tendencia que han seguido en los últimos años. Generar indicadores basados en la tipología descrita en la ilustración 1 permite observar cómo se ha comportado el nivel del componente climático estudiado y su variabilidad a 
través del tiempo. Esto se hace para todas las áreas urbanas de manera mensual. Los datos del municipio se ven representados como puntos en la tipología propuesta y se clasificaron las tendencias en 2 conjuntos diferentes.

- El primer conjunto contiene a aquellas áreas urbanas donde el nivel de la variable ha aumentado (cuadrante II) o disminuido (cuadrante III) pero hay menos volatilidad. Es decir, el clima ha cambiado en comparación a décadas pasadas pero las variables se están consolidando en un nivel estable. La volatilidad en estas regiones se considera como constante en el tiempo ya que, a pesar de los efectos del cambio climático, las condiciones atmosféricas para un mes determinado son parecidas entre sí.

- En el segundo conjunto (cuadrantes I y IV, Ilustracion 1), encontramos a las áreas urbanas que han experimentado cambios de nivel de las variables y que además su variabilidad ha crecido en el tiempo. En el cuadrante I se ubican los municipios que han visto aumentos en el nivel de la variable y además con una volatilidad creciente. Dentro del cuadrante IV se localizan las áreas urbanas que de igual manera presentan en la actualidad más volatilidad en el factor climático pero que, en contraste, el valor de ese factor ha presentado caídas en el nivel promedio.
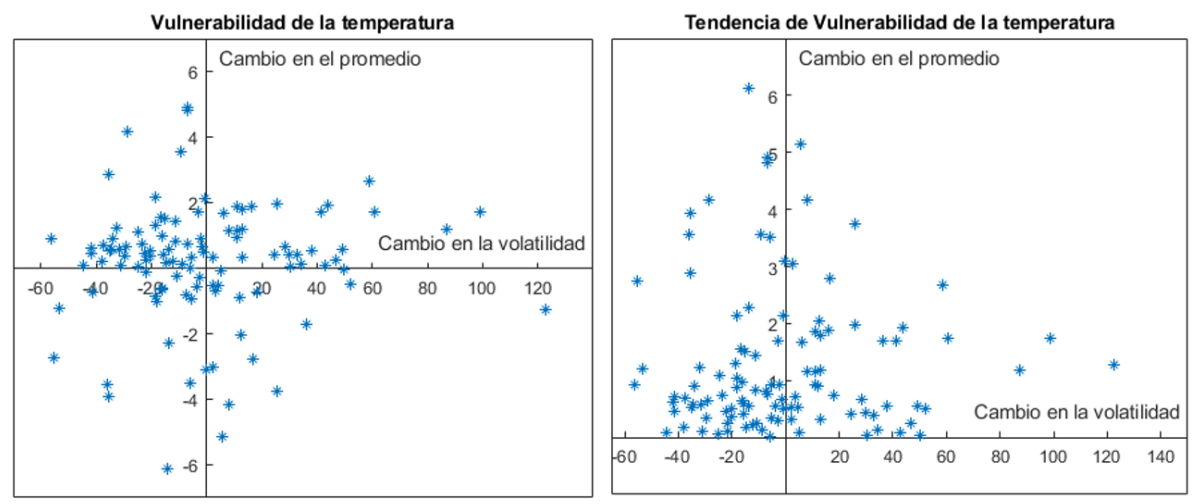

ILUSTRACIÓN 1

Gráficas de la tendencia de temperatura de las áreas urbanas analizadas.

Fuente: elaboración propia con base en RCCDP, y CCSM4_rcp85

Es necesario y razonable suponer que los seres humanos preferimos vivir en lugares cuyo clima es predecible. Desde este punto de vista, el desempeño óptimo de las variables meteorológicas evaluadas se encuentra en el origen de la gráfica, es decir donde el municipio no ha experimentado cambios ni en nivel ni en variabilidad. Entonces decimos que aquellos municipios que se encuentran alejados de las coordenadas $(0,0)$ o del origen han presentado cambios porcentuales más abruptos a lo largo del tiempo (IMCO, 2020).

Debido a la gran diversidad de climas y condiciones atmosféricas observables en el territorio analizado se considera que un aumento o disminución de las variables evaluadas es igualmente perjudicial. Para analizar el cambio climático es poco relevante conocer si la variable climática está cambiando de

forma negativa o positiva. El índice busca medir la volatilidad sin tomar en cuenta la tendencia que ha seguido (en promedio) la temperatura o la precipitación.

Es por esta razón que para el cálculo del indicador se ha decidido incluir una simplificación en el modelo que permitirá, posteriormente, evaluar a todas las áreas urbanas, sin importar sus condiciones atmosféricas históricas. Es decir, se podrá comparar áreas urbanas que tienen una predominancia de temperaturas bajas con otros territorios donde es claro que sus antecedentes históricos los sitúan como ciudades cálidas. Se podría afirmar que la metodología es relativamente indiferente entre el aumento y la disminución de las temperaturas en promedio. En contraste, la varianza es un factor medular del análisis. La simplificación antes mencionada provoca un ligero cambio en la presentación gráfica vista en la ilustración 1 , tanto en los datos y la tipología de vulnerabilidad. Ahora solo se utilizarán dos cuadrantes y no cuatro.

Las localidades que reportaron un aumento o descenso en el nivel de la variable climática están ahora, sin distinción de tendencia, en los cuadrantes I y II. Lo anterior debido a que los datos negativos han sido 
transformados, utilizando el valor absoluto del cambio porcentual, dejando fuera el efecto que el signo pueda tener en las interpretaciones. Las áreas urbanas ya no son catalogadas en 4 regiones, sino que ahora son solo dos clasificaciones las que utilizamos.

Cuadrante I: el área urbana presento un cambio de nivel y al mismo tiempo su volatilidad es mayor en comparación a décadas anteriores. Estas áreas urbanas presentan evidencia de cambio climático y además son menos predecibles las condiciones meteorológicas. En general estas comunidades presentan una vulnerabilidad creciente.

Cuadrante II: la localidad presentó un cambio de nivel en la variable climática pero su volatilidad está descendiendo relativamente. Existe evidencia de un cambio climático en la región de análisis, pero a diferencia del grupo anterior, estas áreas urbanas presentan convergencia hacia cierto nivel en sus variables meteorológicas y atmosféricas. Las poblaciones que se encuentran en este cuadrante se clasifican como de vulnerabilidad constante.

Siguiendo con la lógica que supone mayores efectos de cambio climático en aquellas áreas urbanas más alejados del origen, es posible proponer un método de medición de la vulnerabilidad ante cambios meteorológicos en la zona geográfica. Existen algunas notas importantes:

1) Los puntos en el diagrama de dispersión (localidades) más alejados del origen de los ejes cartesianos son aquellos que han experimentado un cambio más significativo en las variables climáticas, por ello son más vulnerables ante el fenómeno global de cambio climático.

2) Si el área urbana se encuentra en el cuadrante I, en el cálculo del índice, se considera que es doblemente vulnerable, debido a que no solo ha sufrido cambios en clima, también ha experimentado una volatilidad mayor.

3) En el cuadrante II las localidades son clasificados como vulnerables, pero no volátiles y con clima relativamente predecible, esto las coloca en términos comparativos como menos vulnerables que aquellas ubicadas en el cuadrante I.

$d=\sqrt{\left(x_{i}-x_{0}\right)^{2}+\left(y_{t}-y_{0}\right)^{2}}$

Tomando en cuenta las notas anteriores, se utilizó la distancia geométrica entre dos puntos para medir que tan cambiante ha sido la tendencia (Ruiz, 2014). La fórmula utilizada es la cartesiana de distancia:

Donde $(\# \mathrm{i}, \# \mathrm{i})$ son las coordenadas que denotan el cambio porcentual en variabilidad y nivel respectivamente. Las coordenadas con las que se quiere comparar son $(0,0)$, entonces la fórmula final se expresa de la siguiente forma:

$d=\sqrt{\left(x_{i}\right)^{2}+\left(y_{i}\right)^{2}}$

(4)

La medición de la distancia de cada punto, que representa una localidad o área urbana, al origen dio lugar a un indicador de la volatilidad climática, que arroja información por cada mes del año. El indicador tiene ciertas características deseables:

- Es útil para comparar a todas las áreas urbanas.

- Incorpora información sobre el cambio de nivel y variabilidad del clima.

- Presenta un valor óptimo $(\mathrm{d}=0)$ que sirve como parámetro para evaluar el desempeño del clima en las regiones.

- El método es replicable a partir de datos simples.

El método utilizado tiene una limitación importante:

- Es incapaz de distinguir tendencias en los cambios de nivel del clima. El modelo no permite conocer si un municipio es más cálido o frío, seco o lluvioso en comparación con años anteriores.

Para dar solución a este problema fue necesario crear etiquetas que clasificaran a los municipios de acuerdo con las características climatológicas cualitativas que los diferencian del resto de las ciudades. Las etiquetas se definen de la siguiente manera: 1,2, y 3 


\begin{tabular}{|l|l|}
\hline Tipos de Área & Tendencia \\
\hline Áreas urbanas cálidas & $\begin{array}{l}\text { si la localidad ha presentado un aumento de la temperatura en más de } 6 \text { meses al } \\
\text { año. }\end{array}$ \\
\hline
\end{tabular}

\begin{tabular}{|l|l|}
\hline Tipos de Área & Tendencia \\
\hline $\begin{array}{l}\text { Áreas urbanas } \\
\text { lluviosas }\end{array}$ & $\begin{array}{l}\text { Si la región ha experimentado niveles superiores de } \\
\text { lluvia en al menos } 6 \text { meses del año en comparación a } \\
\text { años pasados. }\end{array}$ \\
\hline $\begin{array}{l}\text { Áreas urbanas } \\
\text { secas }\end{array}$ & $\begin{array}{l}\text { Si la tendencia de precipitación es a la baja en } \\
\text { comparación a décadas anteriores. }\end{array}$ \\
\hline
\end{tabular}

En forma similar, es posible saber que tan volátil o estable es un municipio con las siguientes etiquetas: 3

En forma similar, es posible saber que tan volátil o estable es un municipio con las siguientes etiquetas:

\begin{tabular}{|l|l|}
\hline Tipos de Área & Tendencia \\
\hline localidad estable & Si la variable evaluada está consolidando su volatilidad en más de 6 meses. \\
\hline localidad volátil & $\begin{array}{l}\text { Sí en más de } 6 \text { meses del año el área urbana ha experimentado aumentos en la vabilidad del } \\
\text { clima. }\end{array}$ \\
\hline
\end{tabular}

Necesitamos estos indicadores para poder clasificar a los municipios de acuerdo con su tendencia de vulnerabilidad en temperatura y precipitación que han seguido en las tres décadas que estamos analizando. Como vemos los cálculos son para dos periodos de tiempo por lo que el análisis se hizo tomando como base el periodo de 1971-1980 y comparándolo con el periodo de 2001-2010 y después este se comparó con el de 2061-2070.

La medición de la distancia de cada punto, que representa una localidad, al origen dio lugar a un indicador de la vulnerabilidad climática, que arroja información por cada mes del año de donde son las coordenadas que denotan el cambio porcentual en variabilidad y nivel respectivamente. Las coordenadas con las que se quiere comparar son que es el origen. Este punto significa que no hay cambios en la variabilidad o sea que es el punto óptimo.

$T V:=$ Índice de tendencia de volatilidad.

$T V=\sqrt{\Delta \operatorname{Des}^{2}+P_{0}^{2}}(6)$

Este indicador mide los cambios en la volatilidad para cada mes del año.

Presenta un valor óptimo $(T V=0)$ que sirve como parámetro para evaluar el desempeño del clima en las regiones. Es decir, mientras más pequeño el índice de tendencia de volatilidad, menor la volatilidad de la variable analizada en un área urbana en particular tabla 1.

\section{Resultados}

Tabla 1 
TABLA 1.

Índice de tendencia de volatilidad (TV)

\begin{tabular}{lll}
\hline Periodo base & Comparar & Índice de tendencia de volatilidad (TV) \\
$1971-1980$ & $2001-2010$ & 127 máx. \\
$1971-1980$ & $2061-2070$ & 177 máx.
\end{tabular}

Fuente: elaboración propia con base en RCCDP, y CCSM4_rcp85

La tabla 1 muestra un aumento de volatilidad en la región, y con ello de consecuencias poblacionales, sociales, de infraestructura, de recursos y de calidad de vida. Los incrementos en la volatilidad ya son evidentes para el análisis de la línea base de todas las áreas urbanas de la región. Si observamos, la ilustración 2, la dinámica las variables climáticas han ampliado su rango de variabilidad en todas las estaciones en prácticamente todas las ciudades analizadas, es decir, observamos mayor calor y menores lluvias en la canícula entre los meses de marzo a mayo y mayores lluvias y temperatura extremas en el invierno. Cabe recordar que en un ideal de estabilidad se esperaría

que la dinámica de las variables no tuviera cambios significativos alrededor de la media, es decir, la distancia tendría valores cercanos al origen.

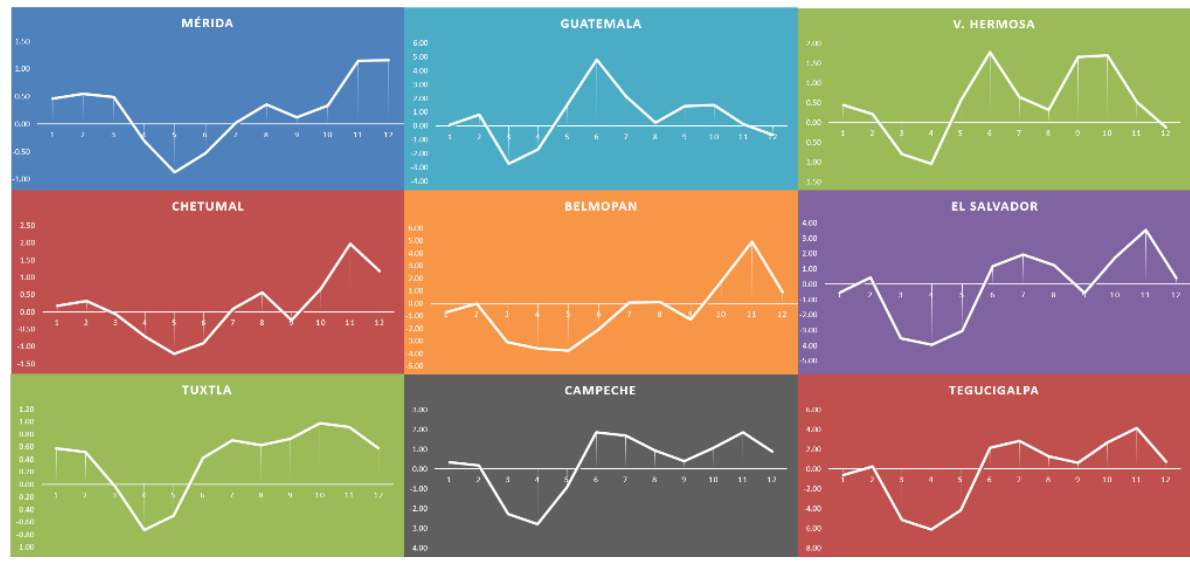

ILUSTRACIÓN 2

Representación gráfica de la tendencia de volatilidad de cada municipio Fuente: elaboración propia con base en RCCDP, y CCSM4_rcp85

Así la ilustración 3, nos muestra dos dinámicas de comportamiento, en color verde el índice de volatilidad del año base y el color café el índice de volatilidad de escenario climático futuro. Si seguimos la línea verde vemos que la región en su conjunto muestra actualmente un incremento en la volatilidad de las variables analizadas, ciudades como Villa hermosa y Belmopán presentan altos índices de volatilidad respecto al año base del estudio, mientras que las ciudades del Salvador, Mérida, Campeche y Tegucigalpa muestran un comportamiento más estable en los años de análisis.

ilustración 3 


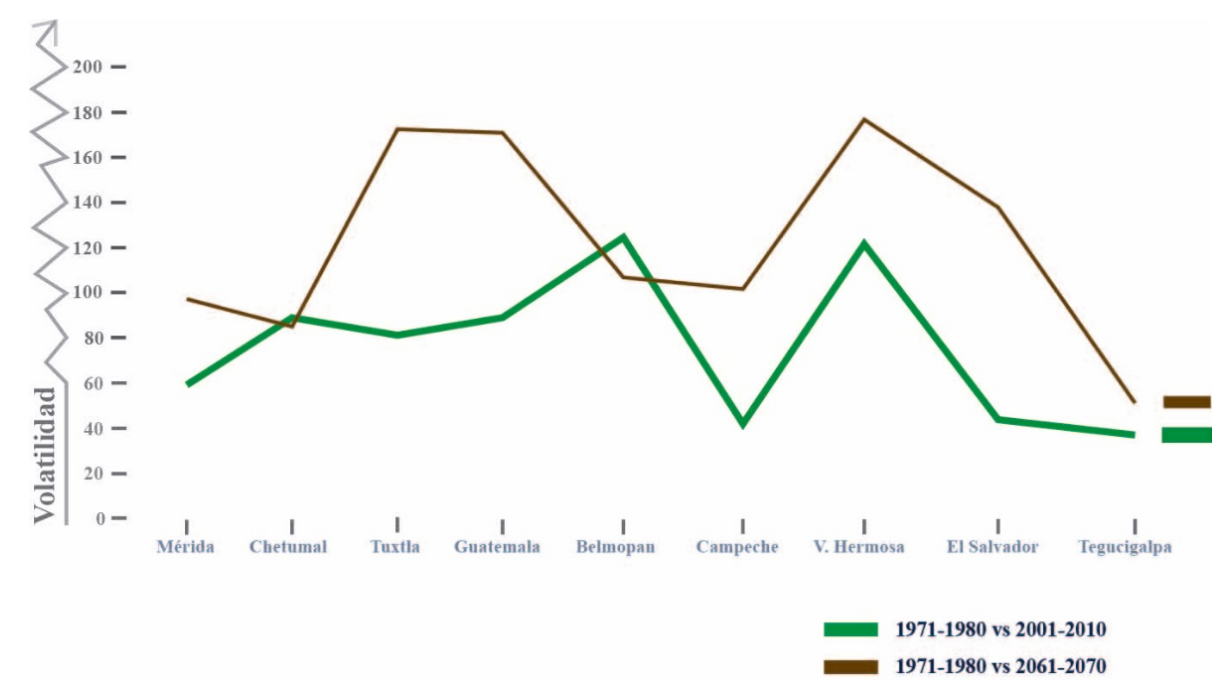

ILUSTRACIÓN 3.

Representación gráfica de la tendencia de volatilidad de cada municipio.Fuente: elaboración propia con base en RCCDP, y CCSM4_rcp85

Tal como se observa en la tabla 2, si aplicamos el análisis de categorías a la variabilidad y volatilidad climática, según la metodología propuesta en la sección anterior, se observa que los primeros años del siglo veintiuno han sido más húmedos y cálidos, aunque existen algunas regiones con condiciones más secas y frías como Belmopán y Campeche. Mientras que los escenarios futuros para mediados del siglo muestran que en términos generales la región de análisis tiene tendencias a desarrollar dinámicas climáticas más secas y caliente; a excepción de la ciudad de Chetumal que aún conserva una tendencia de clima húmedo por su ubicación en la desembocadura del rio Hondo.

Estos incrementos de volatilidad y cambios en las tendencias climatológicas, si bien son generales en toda la región, se pueden analizar en forma puntual para cada una de las áreas urbanas consideradas en el estudio. Por lo que se escogieron como casos de análisis la ciudad de Mérida, por tener un incremento en la volatilidad menor, y el área urbana de Villa Hermosa, por presentar pico de volatilidad tanto en el análisis de línea base como en el análisis de escenarios futuros.

TABLA 2

Tendencias Climatológicas Áreas Urbanas

\begin{tabular}{|c|c|c|c|c|}
\hline \multicolumn{3}{|c|}{$\begin{array}{l}1971-1980 \text { vs } 2001-2010 \\
\text { Tendencias climatológicas Areas Urbanas }\end{array}$} & \multicolumn{2}{|c|}{$1971-1980$ vs $2061-2070$} \\
\hline Clave INEGI & Temperatura & Precipitación & Temperatura & itación \\
\hline MÉRIDA & CÁLIDA & HÚMEDO & CÁLIDA & SECO \\
\hline CHETUMAL & CÁLIDA & HÚMEDO & CÁLIDA & HÚMEDO \\
\hline TUXTLA & CALIDA & HÚMEDO & CÁLIDA & SECO \\
\hline GUATEMALA & CÁLIDA & HÚMEDO & CÁLIDA & SECO \\
\hline BELMOPAN & FRÍA & HÚMEDO & CÁLIDA & SECO \\
\hline CAMPECHE & CALIDA & SECO & CÁLIDA & SECO \\
\hline V. HERMOSA & CÁLIDA & HÚMEDO & CÁLIDA & SECO \\
\hline EL SALVADOR & CÁLIDA & SECO & CÁLIDA & SECO \\
\hline TEGUCIGALPA & CALIDA & $\mathrm{SECO}$ & CÁLIDA & SECO \\
\hline
\end{tabular}

Fuente: elaboración propia con base en RCCDP, y CCSM4_rcp85

La ilustración 4 muestra la volatilidad de la precipitación en ambas ciudades, en donde se presenta un descenso considerable de las lluvias en los escenarios futuros, existiendo un cambio evidente en la temporada 
de lluvias lo que manifiesta eventos extremos a futuro, es decir se esperan sequias más prolongadas, pero con lluvias esporádicas cuantiosas. En el caso de Mérida este comportamiento se realizaría durante la estación de verano y otoño al finalizar la temporada de huracanes.

Con relación al análisis de la variable temperatura, la dinámica de volatilidad en ambas ciudades muestra un incremento de la temperatura en todas las estaciones del año. En el caso, específico de Mérida se espera este comportamiento en el invierno y durante los meses de verano y otoño para el caso de Villa Hermosa. El índice de volatilidad del análisis del año base muestra incrementos en la incertidumbre del comportamiento de la temperatura estando por encima del nivel de estabilidad en ambas ciudades. Al realizar la simulación con los escenarios futuros, se observa que la volatilidad está muy por encima del comportamiento normal de la variable temperatura. Ilustracion 5

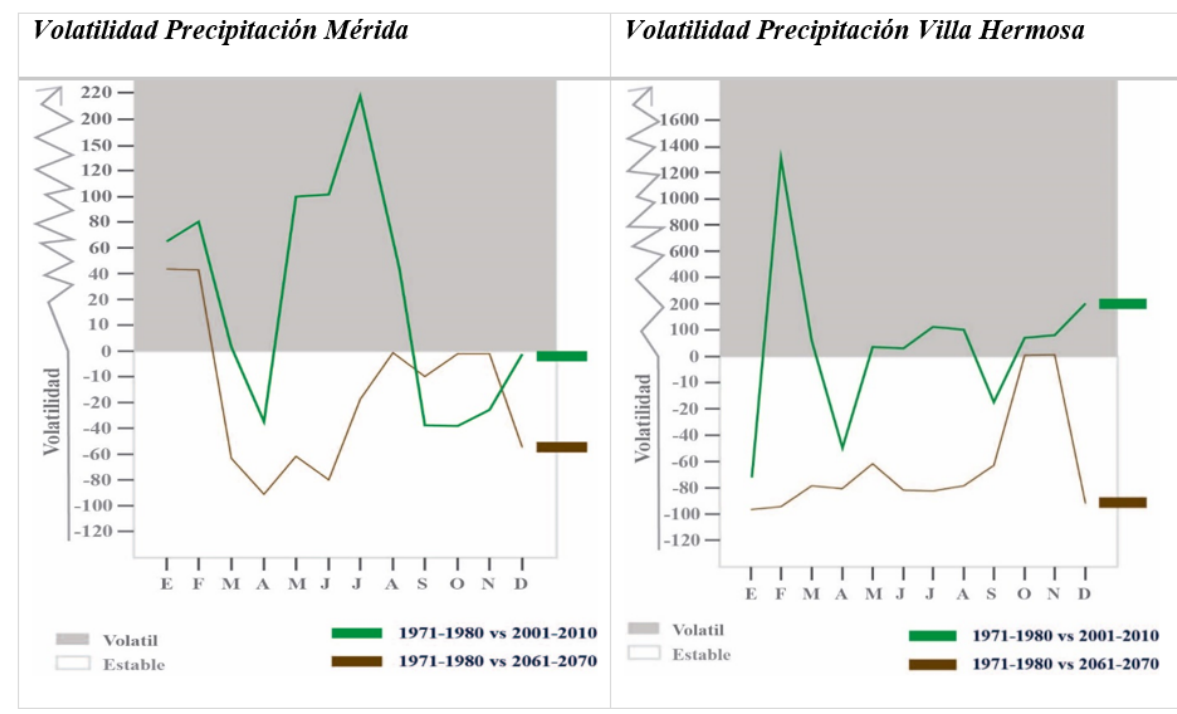

\section{ILUSTRACIÓN 4}

Volatilidad en la precipitación de las áreas urbanas de Mérida y Villa Hermosa. Fuente: elaboración propia con base en RCCDP, y CCSM4_rcp85 Volatilidad Precipitación Mérida Volatilidad Precipitación Villa Hermosa

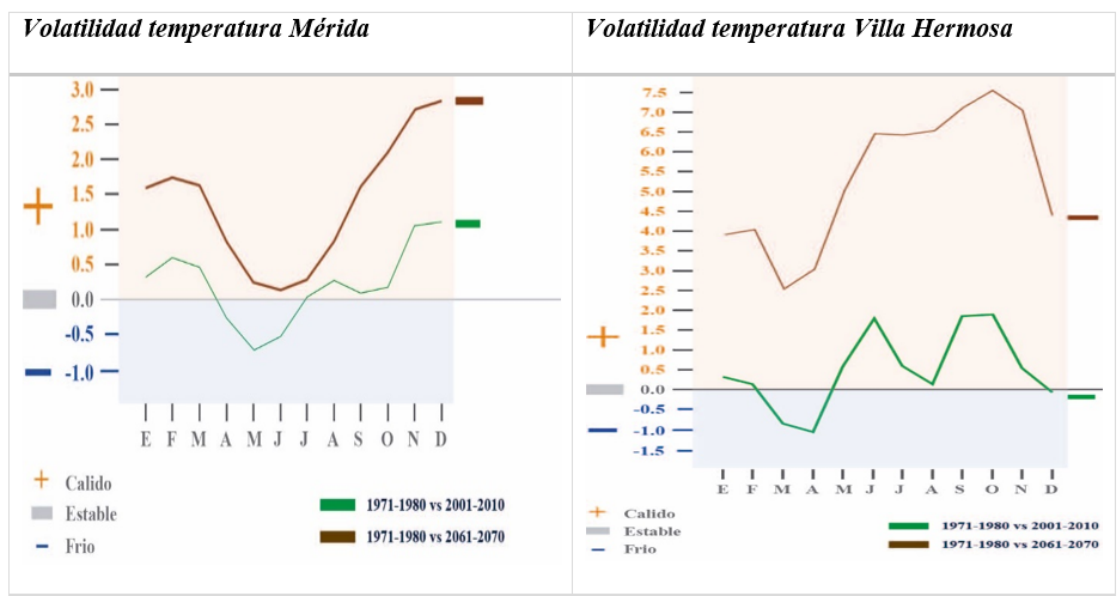

\section{ILUSTRACIÓN 5}

dinámica del índice de volatilidad de la temperatura áreas urbanas de Mérida y Villa Hermosa.Fuente: elaboración propia con base en RCCDP, y CCSM4_rcp85 


\section{Discusión}

La metodología muestra en forma didáctica y sucinta el posible comportamiento de la volatilidad de las variables climáticas en una región o área urbana, usando para ello series temporales de información climática relativamente cortas. Es decir, con una serie de 20 años de base y escenario futuros es posible vislumbrar como se comportará una variable, poniendo en relieve si este la tendencia será seca y húmeda o fría y calurosa. Como se observó en los resultados, las dinámicas locales pueden dar sorpresas en las dinámicas esperadas y mostrar regiones donde el cambio climático tendrá mayores o menores impactos. En resumen, el estudio nos permitió identificar los siguientes impactos para las áreas urbanas analizadas, tabla 3.

TABLA 3.

Impactos del cambio climático en áreas urbanas

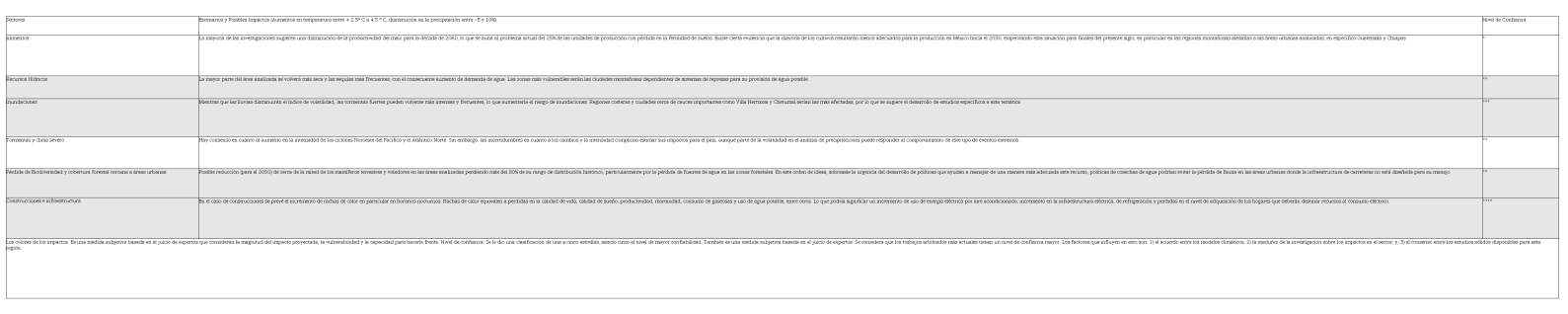

Fuente: elaboración propia con base en (IPCC, 2011) y el análisis de (Álvarez y Blanco, 2016)

\section{Conclusiones}

La región de análisis, es decir, la región sur y sureste de México y la parte norte de centro América, es indudablemente una de las regiones más amenazadas por contingencias naturales: prácticamente todas las regiones urbanas están expuestas a sequias, ciclones e inundaciones. Lamentablemente la sola declaración de esta

exposición no es suficiente para que se tomen medidas pertinentes a la realidad de cada una de las áreas urbanas y localidades analizadas.

Sin embargo, usando una herramienta estadística matemática muy sencilla -el concepto de distancia a un rango medio y su desviación y volatilidad en función de dicho rango medio- nos permite vislumbrar la tendencia climática; y de esta manera observar si el área urbana o localidad analizada se aleja de las temperaturas históricas. Por otro lado, la metodología propuesta, nos ayuda a declarar si la tendencia en volatilidad de la precipitación genera un clima más seco o húmedo de la media histórica y si la dinámica de la volatilidad en la temperatura genera un clima más cálido o frio.

Esta descripción permite matizar otros estudios y darles un contexto más didáctico y de fácil aplicación para la toma de decisiones en política pública, en particular en las áreas urbanas donde la tendencia a climas secos y cálidos genera una serie de consecuencias que deben ser analizadas en conjunto, como la necesidad de diversificar las fuentes de agua potable y generación hidroeléctrica, ver los efectos de las rachas de calor en la calidad de vida de la población, la calidad de sueño y productividad y en última instancia en el consumo de niveles de energía destinados a aire acondicionado o refrigeración superiores a los niveles actuales.

\section{LITERATURA CITADA}

Álvarez, A., \& Blanco, J. (2016). Variabilidad y Cambio Climático. Impactos, Vulnerabilidad y Adaptación al Cambio Climático en América Latina y el Caribe. Ciudad de México: UNAM. 
Hardoy, J., \& Lankao, P. R. (2011). Latin American cities and climate change: challenges and options to mitigation and adaptation responses. Current Opinion in Environmental Sustainability, 158-163. https://doi.org/10.1016/ j.cosust.2011.01.004

Heard, C. L., \& Olivera, S. M. (2013). Evaluación económica de la resistencia térmica de la vivienda de interés social en las ciudades tropicales de México. . Acta Universitaria, 17-29. https://doi.org/10.15174/au.2013.458

Instituto Mexicano de Competitividad. (6 de enero de 2020). https://imco.org.mx/. Obtenido de https:// imco.org.mx/wp-content/uploads/2012/7/boletindeprensa_ivc_final.pdf

Olivera, S. (2019). La administración del riesgo como instrumento de desarrollo. La Paz: Banco Mundial.

Ruiz, J. (2014). Geometría analítica. Mexico: Grupo Editorial Patria.

Satterthwaite, D. (2009). The implications of population growth and urbanization for climate change. Environment and Urbanization, 545-567. https://doi.org/10.1177/0956247809344361

Climate Vulnerability Monitor 2nd Edition. (6 de enero de 2012). www.daraint.org/cvm2/method. Obtenido de www.daraint.org: www.daraint.org/cvm2/method

DARA. (2012). Climate Vulnerability monitor-A guide to the cold calculus of a hot planet. Madrid, Spain.: Climate Vulnerable Forum.

Figlewski, S. (1997). Forecasting volatility. Financial markets, institutions \& instruments, 1-88. https:// doi.org/10.1111/1468-0416.00009

IPCC. (2011). special report on renewable energy sources and climate change mitigation. Geneve: IPCC.

Loa, J., Cabos, W., Álvarez García, F., \& Ruiz de Elvira, A. \&. (2006). . Análisis preliminar de la volatilidad de extremos de precipitación en la Península Ibérica. https://repositorio.aemet.es/.

Martinez Gonzalez, C. A. (2015). Percepción pública del cambio climático en México. Universidad Iberoamericana Ciudad de México. Departamento de Comunicación.

Oglesby, R., Rowe, C., Grunwaldt, A., Ferreira, I., Ruiz, F., Campbell, J., \& Lopez, P. (2016). A high-resolution modeling strategy to assess impacts of climate change for Mesoamerica and the Caribbean. American Journal of Climate Change, 202-2. https://doi.org/10.4236/ajcc.2016.52019

Olivera, S. M. (2017). Opciones de política para transitar hacia una senda de desarrollo baja en carbono: México. Sucre: USFXC.

REGIONAL CLIMATE CHANGE CONSORTIUMFOR LATIN AMERICA AND THE CARIBBEAN. (25 de marzo de 2020). http://rccdp.unl.edu/portal/old/data/DataDownload.html. Obtenido de http://rccdp.unl.edu/portal/old/data/DataDownload.html: http://rccdp.unl.edu/portal/old/ data/DataDownload.html 